\title{
Design of light-sheet fluorescence imaging platform for axon tracing over entire brains
}

Kevin Takasaki $^{1}$, Ruixuan Gao ${ }^{2}$, Emily Turschak ${ }^{1}$, Michael J. Taormina $^{1}$, Philip R. Nicovich ${ }^{1}$, R. Clay Reid ${ }^{1}$ ${ }^{1}$ Allen Institute for Brain Science, Seattle, WA, USA. ${ }^{2}$ MIT McGovern Institute for Brain Research, Cambridge, MA, USA

\section{Abstract Text}

Light-sheet fluorescence microscopy combined with tissue expansion enables imaging thick tissue sections with sub-100 nm effective resolution at acquisition speeds exceeding 100 Megavoxels/second [1]. We are seeking to apply this combination of techniques to the complete mapping and tracing of individual axons over entire mammalian brains, an approach we refer to as Axonal Connectomics. The initial scale of this effort entails acquiring $200 \mathrm{~nm}$ voxels over 10 $\mathrm{cm}^{3}$ of tissue, comprising $\sim 1.25 \mathrm{~PB}$ of raw imaging data to be stored, processed, and analyzed, and at a rate of 100 Megavoxels/second would require roughly 4 years of continuous imaging.

Here, we describe our approach to meeting the simultaneous optimization of image quality, speed, and reliability in the design of our prototype light-sheet imaging platform. Given the high demands on resolution and acquisition speed, we have alternated between a 1.0 NA multi-immersion objective and a 1.1 NA water immersion objective for detection, positioned at an angle roughly 60 degrees from the horizontal, with a sheet angle of roughly 30 degrees to be able to accommodate a high-NA objective in a light-sheet geometry. For a practical illustration, a $50 \mu \mathrm{m}$ thick tissue section expanded 2times to $100 \mu \mathrm{m}$ requires a sheet length (field-of-view) of $200 \mu \mathrm{m}$, placing our method firmly in the challenging position of having to push constraints on the traditional trade-offs between field-of-view, resolution, and speed, all at once. A Gaussian sheet with a field-ofview (Rayleigh length) of $200 \mu \mathrm{m}$ implies a beam waist of roughly 3 
$\mu \mathrm{m}$ FWHM, an order of magnitude larger than the target resolution. With a high-NA detection objective, the axial resolution will be determined principally by the collection NA; however, when the thickness of the sheet exceeds the depth-of-field of imaging, the problem of resolution is converted to a problem of contrast in densely labeled samples, as is the case for fiber bundles in white matter. Manipulating the expansion factor provides some flexibility in tuning optical trade-offs, but changing the size of the tissue implies changing imaging parameters as well.

A number of studies have analyzed the performance of various configurations of light-sheet fluorescence microscopes in theory and in practice. A recent numerical analysis by Remacha, et al. systematically compared imaging performance in terms of two relevant quantities: axial resolution defined by the distance along the detection axis where intensity first drops to a fraction of peak, and image contrast defined by the total fluorescence generated by the light sheet within a certain distance from the detection focal plane [2]. We have expanded on their analysis of resolution and contrast with two additional systematic considerations: the contribution of the collection NA, as well as comparison of timeaveraged sheet versus confocal slit-scanning. In our presentation, we will describe the results of our theoretical analysis generally and implications for our imaging of axons over large volumes, as well as exhibit data from both imaging standards and immuno-stained tissue samples for axon tracing. 